\title{
Modelling Visual Appearance of Handwriting
}

\author{
Angelo Marcelli, Antonio Parziale, and Adolfo Santoro \\ Natural Computation Laboratory, \\ DIEM, University of Salerno \\ Via Ponte Don Melillo, Fisciano (SA), Italy \\ \{amarcelli, anparziale, adsantoro\}@unisa.it \\ http://nclab.diiie.unisa.it
}

\begin{abstract}
We present an experimental validation of a model of handwriting style that builds upon a neuro-computational model of motor learning and execution. We hypothesize that handwriting style emerges from the concatenation of highly automated writing movements, called invariants, that have been learned by the subject in correspondence to the most frequent sequence of characters the subject is familiar with. We also assume that the actual shape of the ink trace contains enough information to characterize the handwriting style. The experimental results on a data set containing genuine, disguised, and forged (both skilled and naive) documents show that the model is an effective tool for modeling intra-writer and inter-writers variability and provides quantitative estimation of the difference between handwriting styles that is in accordance with the difference in the visual appearance of the handwriting.
\end{abstract}

Keywords: handwriting learning and generation model, handwriting shape description, handwriting analysis.

\section{Introduction}

Handwriting analysis, which requires the detection and examination of distinctive features within the ink traces representing the words, provides a valuable help in several research fields. In medical field, handwriting analysis provides a useful complement to other clinical investigations in diagnosing many movement disorders, such as Parkinson's disease. In forensics, the examination of particular characteristics of the ink trace allows the expert to evaluate the authenticity of a handwritten text. Handwriting recognition, which allows optimizing the handling of manuscript documents, requires the detection of distinguishing features for interpreting the characters the ink trace represents. Since any phenomenon can be better understood and analyzed when the generative process is known, investigating the process that underlies handwriting might give some guidelines for handwriting analysis. Investigations in motor control have shown that handwriting is performed by properly coordinating movements of the lower arm, wrist and fingers, which give rise to the sequence of elementary shapes (i.e. the strokes) whose concatenation generates the ink trace representing the characters and, ultimately, the word. Fluency, then, emerges when these movements are executed

A. Petrosino (Ed.): ICIAP 2013, Part II, LNCS 8157, pp. 673-682, 2013.

(C) Springer-Verlag Berlin Heidelberg 2013 
in such a way to minimize the writer's energy consumption 122. It has also been shown that at the beginning of handwriting learning, each stroke aimed at reaching the target point that has been visually selected is executed independently from the previous or the following one. By repeated practice, the sequence of target points becomes familiar to the writer, as well as the sequence of motor commands needed to reach them, so that the next movement can start before the current one terminates. When the learning completes, fluency is achieved, in that both sequences have been learned and stores in such a way that they are resorted from memory and the corresponding movements executed automatically with proper timing and without any visual and proprioceptive feedback, as it were an elementary movement 34 . We call these highly automated sequences invariants, in that they are used every time the word to be written includes sequence of characters for which the corresponding writing movements have been learned. From the definition of invariants and from the paramount role played by repeated practice during handwriting learning, it follows that the sequence of characters for which an invariant is developed depends, apart from the cognitive and the motor skills of the writer, from the lexicon he is familiar with. Thus, handwriting production may be thought of a sequential concatenation of the invariants, each one corresponding to a sequence of characters and being represented in different cortical regions of the brain, namely the basal ganglia and the cerebellum, as a sequence of target points and a motor plan [5]. Handwriting analysis, similarly, can be seen as a decomposition process that extracts the invariants and estimate the sequence of target points and the motor plan associated to each of them. According to the framework we have sketched above, characterizing handwriting requires to extract the invariants from the handwritten samples and to estimate both the target points and the motor plan. In a previous paper we have conjectured that all those aspects are embedded into the ink trace and can be estimated by looking at the actual shape of the ink, and shown the results of a preliminary experiment to support our claim [6]. In this paper, we address the fundamental question on whether the proposed model is able to effectively estimate both the intra-writer and the inter-writer variability. The results of the experiments provide further support to our model, and also shown that the quantitative difference in the handwritten samples as measured by the model are in accordance with the visual appearance of handwriting styles.

The remaining of the paper is organized as it follows. In Section 2 we briefly summarize the model of handwriting style under investigation, while the procedure for computing the model parameter is described is described in Section 3. Section 4 reports the experiments performed for measuring handwriting variability through the set of feature we have proposed and discuss the obtained results. Eventually, in the conclusion we discuss how and to which extent the experiments corroborate our conjecture and outline the future work. 


\section{Modeling Handwriting Style}

A handwriting style results from the writer motor learning, which is achieved by means of a complex interaction that involves motor planning and motor execution. Motor planning accounts for the relative position of the targets and the amount of anticipation, whereas motor execution accounts for the variations of the actual shape of the trace in different executions depending on the actual conditions of the actuator when the execution starts. Thus, modeling handwriting requires to describe the shape of the strokes and the amount of time superimposition between successive ones 6 .

Many models were proposed in the literature for describing the shape of a stroke, and we have chosen to describe it with an ellipse, using a least-squares technique [7], because this primitive shape holds the kinematics property of handwriting [8].

Given a stroke, its description is provided by estimating the three parameters of the ellipse that best fit the stroke: the half-length of both the major and the minor axis, which are rotation invariant and size dependent, and the angle $\theta$ between the major axis of the ellipse and the x-axis. Because of the slant is a relevant characteristics of the handwriting style whereas size-dependent variations are not, we include in the model only $\theta$ to discriminate among rotated strokes.

In order to estimate the amount of time superimposition between a pair of successive strokes we compute the angle $\gamma$ between the major axis of the ellipses that best fit the strokes. Summarizing, a handwritten trace composed of $\mathrm{N}$ strokes will be associated with the following sequences:

$$
\begin{gathered}
\Theta=\left(\theta_{1}, \theta_{2}, \ldots, \theta_{N}\right) \\
\Gamma=\left(\gamma_{1}, \gamma_{2}, \ldots, \gamma_{N-1}\right)
\end{gathered}
$$

The final model $M$ is achieved by averaging the values of each sequence:

$$
M=\left(\Theta_{\text {avg }}, \Gamma_{\text {avg }}\right)
$$

\section{Model Parameter Estimation}

To measure the model parameter we need to preliminary extract from the trace the sequence of strokes, i.e. to recover the writing order and to locate the segmentation points between successive strokes. In a previous work [9] we have presented an algorithm for writing order recovery which uses local criteria to formulate hypothesis on traversing each intersection, and global search for the final selection. It also incorporates criteria for finding the beginning and the end of each fragment and to deal with movements including pen-lifts but still resulting in a single connected component. The resulting implementation is based on the Fleury's algorithm for graph traversal, incorporates rules derived from handwriting generation modeling for both selecting the beginning and the end of each 
fragment and to deal with the pen-up possibly present, and eventually resorts to good continuity criteria when alternative solutions at intersections are still possible after the previous processing. At the end of this step, thus, the ink of each fragment has been unfolded according to the reconstructed writing order. The strokes are generally hidden in the ink due to both noise and anticipatory effects. The noise originates from several sources, from the digitizer to erratic hand or finger movements. The anticipatory effect originates from time-superimposition of strokes that allows starting a new stroke before the end of the previous one, leading to the fluency of the handwriting movements and the smoothness of the ink. In a previous work [10] we have developed a stroke segmentation algorithm by exploiting the concept of saliency introduced for modeling visual attention shift. Following this approach, the electronic ink represents the scene the system is looking at, and its curvature represents the feature whose saliency is estimated. Thus, segmentation points correspond to the highest values of the saliency map. The obtained segmentation is much more invariant with respect to locally prominent but globally non-significant changes of curvature and compares favorably even with those exploiting changes in the writing speed as they are available in the on-line case. Given the sequence of strokes, we describe it with the pair $\left(\Theta_{a v g}, \Gamma_{a v g}\right)$, as described in the previous section.

\section{Experimental Validation}

To verify that the model is able to capture the differences between handwriting styles we used it for computing the intra-writer and inter-writer variability of some documents extracted by a data set collected during a nationwide experiment to assess the performance of FDEs [11. Some of the subjects were requested to produce a disguised handwriting, i.e. a handwriting in which they consciously modify it with respect to their genuine one. None of the subjects was a skilled forger, but they were allowed to practice for 15 minutes before producing the disguised document. For our purposes, we also asked to some skilled forgers to produce a copy of some genuine documents of the data set. The genuine documents produced by the other subjects, moreover, are considered as naive forgeries. Therefore, for each genuine document a disguised, a naive and a skilled version are available.

As explained before, handwriting style refers to the execution of a sequence of strokes that have been previously learned by the writers, so that they are produce automatically and thus fluently. Among them, the movements executed without leaving a trace on the paper, as it happens during pen-up's, are beyond the limits of our analysis. Thus, we consider only the fragments of the words, i.e. the parts of the traces drawn without lifting the pen. Each fragment is still a complex movements, and as such may include subsequences of strokes that have been learned better than other and therefore are executed in a more automated way. For instance, the sequences of strokes appearing in sequences of characters with whom the subject is more familiar with may have been practiced more frequently and therefore their execution is more automated than that of less practiced ones. According to our definition of handwriting style, these sequences, 
we call invariants, are the ones that characterize it better and therefore the ones that should be modeled as in the previous Sections.

The detection of the invariants is achieved by an ink matcher [12 that finds the longest common sequences of strokes with similar shapes between the inks of a pair of fragments. As similarity measure, we adopt the Weighted Edit Distance (WED), which measures the shape similarity between pair of strokes [13. The shape similarity of a sequence is obtained by adding the WED of its strokes. When two fragments $f_{x}$ and $f_{y}$ are sent to the ink matcher, each sequence of strokes corresponding to the detected invariant is described with the pair $\left(\Theta_{\text {avg }}, \Gamma_{\text {avg }}\right)$, then the Euclidean distance $d_{x y}$ between the pairs $\left(\Theta_{\text {avg }}, \Gamma_{\text {avg }}\right)_{f x}$ and $\left(\Theta_{\text {avg }}, \Gamma_{\text {avg }}\right)_{f y}$ is computed. In our experiments, the documents contain the same text, a summary of a children tale well-known to the writers, and we selected a subset of 17 words, so as to include words of different length, representative of the word length distribution in the Italian language. Depending on the subject handwriting style, it may happen that the same words may be split in a different number of fragments, that fragments may corresponds to different sequence of characters or drawn by using different sequence of strokes. To cope with all these sources of variability, we searched for invariant by applying the ink matching between each pair of fragments.

For each word of the genuine document, the fragments were automatically extracted and the detection of invariants was performed for each pair of them. We characterized the intra-writer variability of the genuine document with the normal distribution $N\left(\mu_{g}, \sigma_{g}\right)$ of the Euclidean distances between the pairs of invariants found in the document. For each word of the questioned (i.e. disguised, naive or skilled) document, the fragments were automatically extracted and the detection of invariants between the genuine and the questioned fragments was performed. We characterized the inter-writer variability between the genuine and the questioned document with the normal distribution $N\left(\mu_{q g}, \sigma_{q g}\right)$ of the Euclidean distances of the pairs of invariants found while comparing the two documents. We would expect that the intra-writer variability estimated as above be smaller than the inter-writer variability, but also that the inter-writer variability increases as we compare the genuine with the disguised, the skilled and the naive, respectively.

Figures 14 4] show two words extracted from the genuine document "297", the disguised document "104", the skilled document "297s" and the naive document "990", while the distributions that characterize the intra-writer and inter-writer variability are plotted in Figures 9, 11, Similarly, Figures 5, 8 , show two words extracted from the genuine document "137", the disguised document " 574 ", the skilled document "137s" and the naive document " $567 "$, and the distributions that characterize the intra-writer and inter-writer variability are plotted in Figures 12, 14. Similar trends have been observed for all the words and for all the documents. The plots show that, as expected, the distance between the handwriting style between the genuine and the other documents increases as the disguised, the skilled and the naive are considered. They also show that the difference in handwriting style as it can be estimated on the visual appearance of 

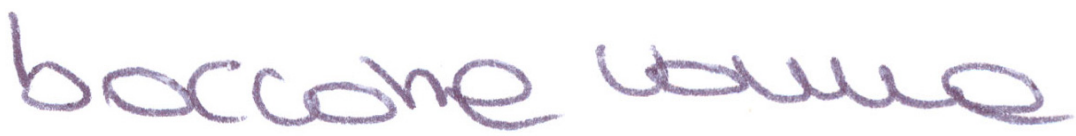

Fig. 1. The words "boccone" and "nonna" extracted from the genuine document 297

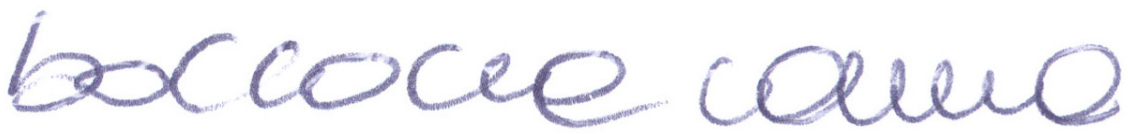

Fig. 2. The words "boccone" and "nonna" extracted from the disguised document 104

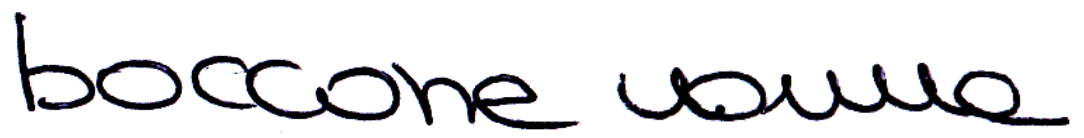

Fig. 3. The words "boccone" and "nonna" extracted from the skilled document 297s

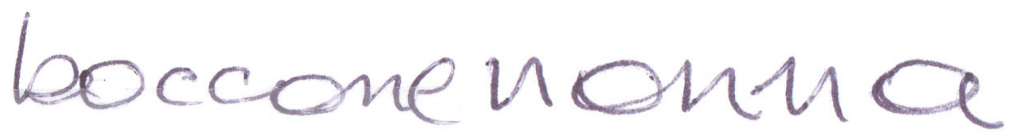

Fig. 4. The words "boccone" and "nonna" extracted from the naive document 990

the traces is reflected in the distance computed as above. For instance, the visual appearance of the words in fig. 1 is closer to those of fig. 2 than to those of fig. 3 and 4. As a matter of fact, the disguising was obtained mainly by slightly changing the slant of the vertical strokes and by changing the visual appearance of the character "n" in the word "boccone". The words from the skilled document, on the other hand, resemble closely the original shape, but not the dynamics, as it can be seen by looking at the character "b" of the word "boccone", that in the genuine is produced without the retracing (or the pen up) occurring between the vertical bar and the loop shaped part of the trace. Eventually, the biggest difference is observed when comparing the genuine with the naive, in that the two writers clearly adopt different styles. The similarities between the difference in the visual appearance and the inter-writer variability measured by our method are even more evident for the documents in fig. 5, 8 .

The ability of the proposed model to capture the distinctive aspects of handwriting style has been tested on a writer identification task performed on the same data set. To reduce as much as possible the influence of the classification strategy on the output, we have considered a very simple decision rule: two documents are considered as produced by the same writer if $\mu_{q g} \leq \mu_{g}$. With such a simple criterion, we were always able to correctly discriminate disguised from skilled and naive forgeries. Furthermore, the differences $\mu_{q g}-\mu_{g}$ show the expected increasing trend as the questioned becomes the disguised, skilled and naive, respectively. 


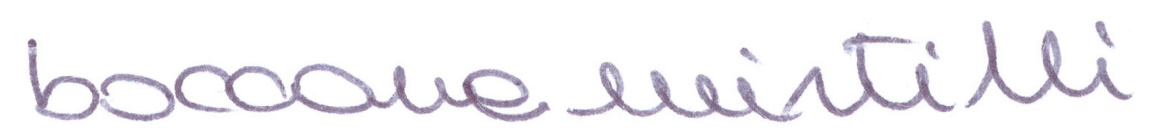

Fig. 5. The words "boccone" and "mirtilli" extracted from the genuine document 137

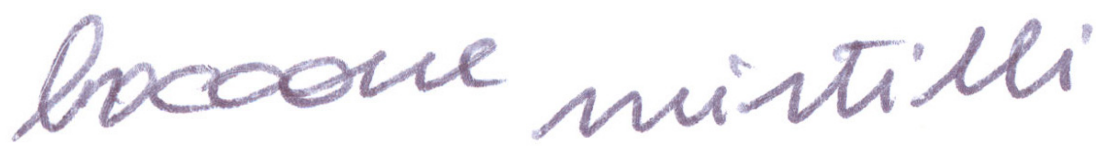

Fig. 6. The words "boccone" and "mirtilli" extracted from the disguised document 574

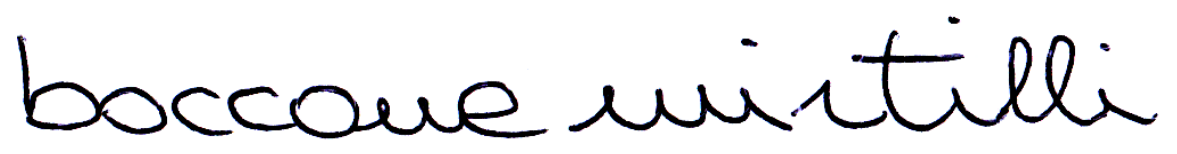

Fig. 7. The words "boccone" and "mirtilli" extracted from the skilled document 137s
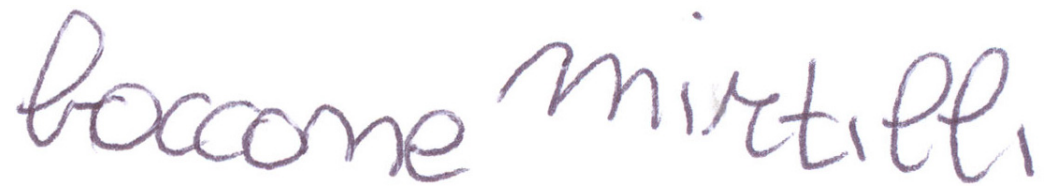

Fig. 8. The words "boccone" and "mirtilli" extracted from the naive document 567

\section{Conclusions}

The experimental results reported in the previous section provide evidence that the model we have suggested is effective in capturing the distinctive aspects of handwriting style and can therefore represent a viable tool for handwriting analysis. They also show that differences in visual appearance of handwriting, on which our intuitive judgment of difference in handwriting styles seems to be based upon, are reflected in quantitative differences of the model parameters. All together, the results provide a further evidence of the link between the neurocomputational model of handwriting learning and execution, that explains how the handwriting styles originate, and our computational model of handwriting style, that estimates the parameters of the system for producing a given style.

The style model we have developed may be useful in many applications. In medical field, it could be used for monitoring the changes of a subject handwriting style along the time. Those changes has been shown to be related to both age and neuro-degenerative diseases [14, and therefore their monitoring may provide early diagnosis signs, as well as indication on the brain regions where the impairment occurs. In forensic document examination, the model of style could be used for writer verification and/or identification, by estimating 


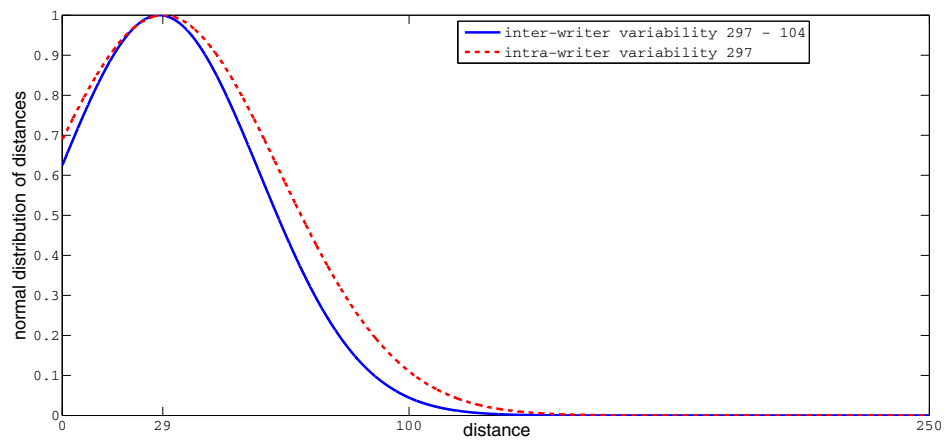

Fig. 9. Inter-writer variability between the genuine (297) and the disguised writing (104)

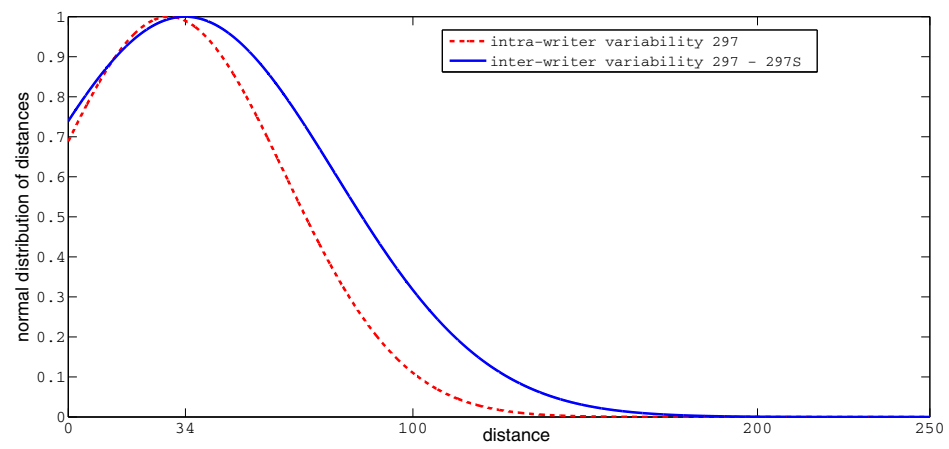

Fig. 10. Inter-writer variability between the genuine (297) and the skilled forger (297S)

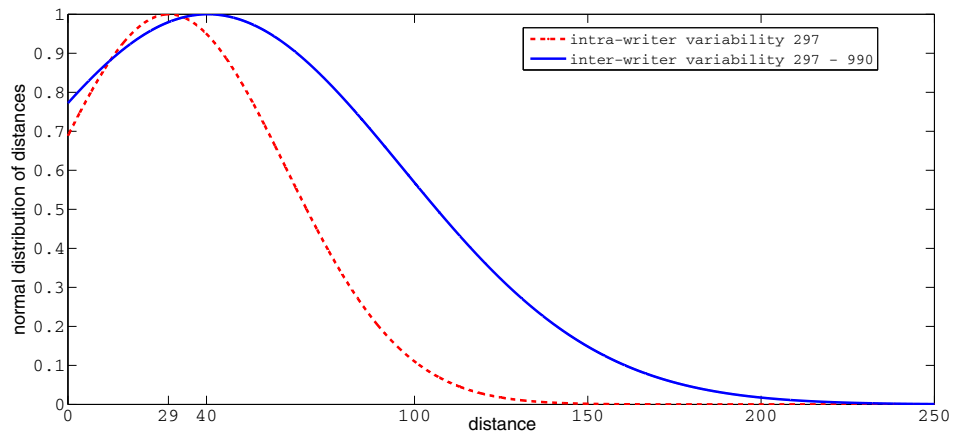

Fig. 11. Inter-writer variability between the genuine (297) and the naive forger (990) 


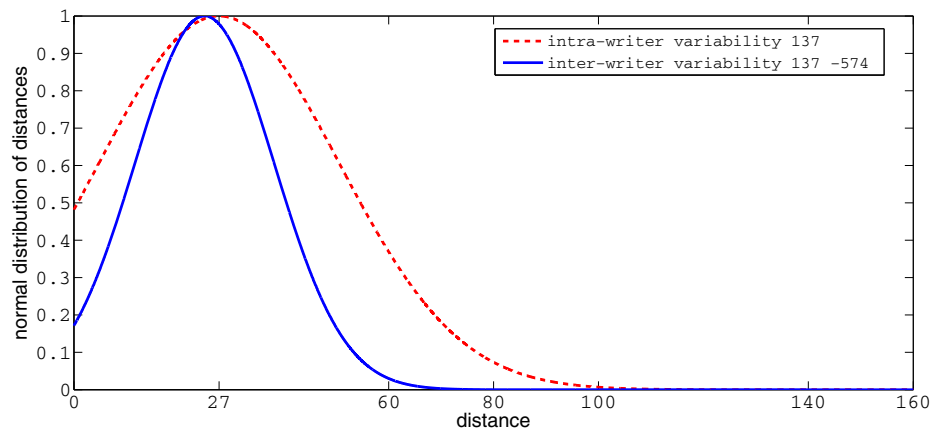

Fig. 12. Inter-writer variability between the genuine (137) and the disguised writing (574)

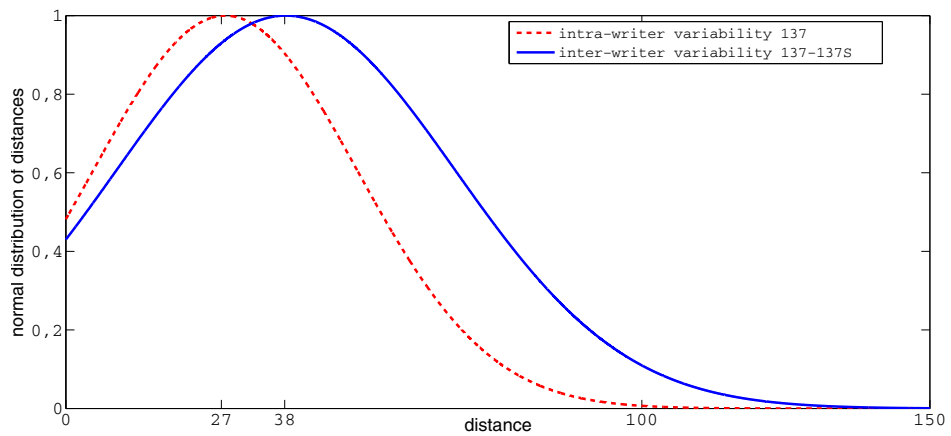

Fig. 13. Inter-writer variability between the genuine (137) and the skilled forger (137S)

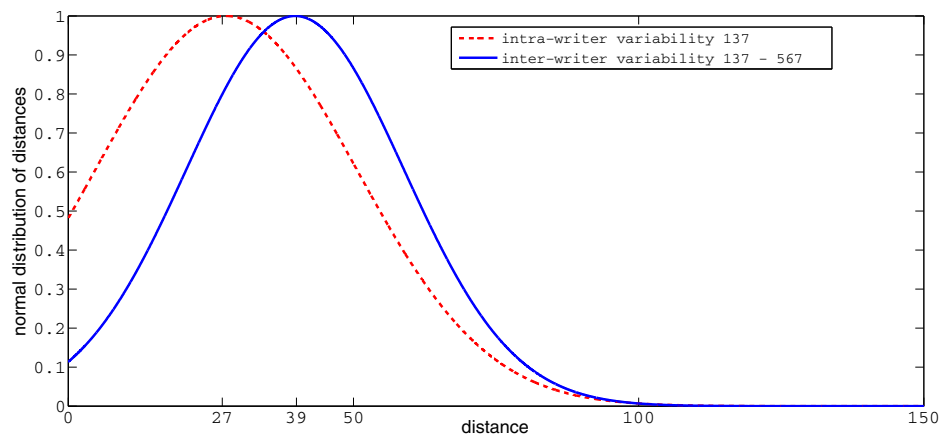

Fig. 14. Inter-writer variability between the genuine (137) and the naive forger (567) 
the intra-writer variability on a genuine document, the inter-writer variability between the questioned document and the genuine and eventually comparing the two, as shown in our simple experiment. In handwriting recognition, the model suggests that features able to describe the shape of the sequence of strokes are the most natural candidates for capturing the distinctive aspects of handwriting.

Applying the model in any of the fields mentioned above, however, requires improving the model in many different ways. So far, the model does not include any explicit description of the actual shape of the stroke, since we use only the orientation of the main axes of the best fitting shape primitive. Taking into account the other parameters of the stroke shape primitives, as well as considering other models of stroke generation, may improve the discriminative power of the model. Similarly, we plan to evaluate different techniques for measuring the similarity between handwriting styles.

\section{References}

1. Thomassen, A.J., Teulings, H.L.: Constancy in stationary and progressive handwriting. Acta Psychologica 54(1), 179-196 (1983)

2. Kawato, M.: Internal models for motor control and trajectory planning. Current Opinion in Neurobiology 9(6), 718-727 (1999)

3. Grossberg, S., Paine, R.W.: A neural model of cortico-cerebellar interactions during attentive imitation and predictive learning of sequential handwriting movements. Neural Networks 13(8), 999-1046 (2000)

4. Senatore, R.: The role of Basal ganglia and Cerebellum in Motor Learning: A computational model. PhD Thesis, University of Salerno (March 2012)

5. Senatore, R., Marcelli, A.: A Neural Scheme for Procedural Motor Learning of Handwriting. In: 2012 International Conference on Frontiers in Handwriting Recognition (ICFHR), pp. 659-664. IEEE (September 2012)

6. Marcelli, A., Parziale, A., Santoro, A.: Modeling Handwriting Style: a Preliminary Investigation. In: 2012 International Conference on Frontiers in Handwriting Recognition (ICFHR), pp. 411-416. IEEE (September 2012)

7. Fitzgibbon, A., Pilu, M., Fisher, R.B.: Direct least square fitting of ellipses. IEEE Transactions on Pattern Analysis and Machine Intelligence 21(5), 476-480 (1999)

8. Bezine, H., Alimi, A.M., Sherkat, N.: Generation and Analysis of Handwriting script with the Beta-elliptic Model. In: Ninth International Workshop on Frontiers in Handwriting Recognition, IWFHR-9 2004, pp. 515-520. IEEE (2004)

9. Cordella, L.P., De Stefano, C., Marcelli, A., Santoro, A.: A New Graph Search Algorithm for Writing Order Recovery. In: ICPR 2010, pp. 23-16 (2010)

10. De Stefano, C., Guadagno, G., Marcelli, A.: A saliency-based segmentation method for online cursive handwriting. International Journal of Pattern Recognition and Artificial Intelligence 18(07), 1139-1156 (2004)

11. Nocentini, C.: Il problema grafologico. In: Giordano, editore (2006) (in Italian)

12. De Stefano, C., Marcelli, A., Santoro, A.: On-line cursive recognition by ink matching. In: Phillips, J.G., Rogers, D., Ogeil, R.P. (eds.) Proceedings of IGS 2007, pp. 23-37 (2007)

13. De Stefano, C., Garruto, M., Lapresa, L., Marcelli, A.: Using strings for on-line handwriting shape matching: A new weighted edit distance. In: Roli, F., Vitulano, S. (eds.) ICIAP 2005. LNCS, vol. 3617, pp. 1125-1132. Springer, Heidelberg (2005)

14. Caligiuri, M.P., Mohammed, L.A.: The neuro science of handwriting: applications for forensic document examination. CRC Press (2012) 